Ann. Biol. anim. Bioch. Biophys., 1978, 18 (3), 695-698

\title{
Influence d'une modification brutale de l'environnement sur l'apparition de l'œstrus chez la lapine nullipare
}

par Brigitte LEFEVRE, B. MORET

Station centrale de Physiologie animale, I. N. R. A.

78350 jouy en Josas, France

Summary. Sudden environmental change as a factor in the onset of estrus in doe-rabbits.

Nulliparous rabbit does 4 to 5 months old kept in individual cages either outside (Group 1) or in a room (Group 2) with a regulated light-dark cycle (12 L : 12 D) were moved into a room with a daylight ratio of $16 \mathrm{~L}: 8 \mathrm{D}$. Receptivity to the male was tested $24 \mathrm{hrs}$ later every day for 4 days. Such a change induces the onset of estrus in the two groups ; the different environmental factors, i. e. daylight ratio, cage, handling and male stimuli, being almost equally effective and acting synergistically. All of these factors may act as stress.

Si la lapine domestique est considérée comme une femelle en œstrus permanent, contrairement aux femelles à cycles œstriens réguliers (Hammond et Marshall, 1925), il semble en réalité exister des périodes d'œstrus plus ou moins longues, séparées par de courtes périodes de diœstrus. Les travaux sur le comportement sexuel dans cette espèce (Myers et Poole, 1962 ; Heath, 1972) ou sur la croissance folliculaire (Hill et White, 1933 ; Smelser et al., 1934) laissent supposer l'existence d'un cycle de 6 à 7 jours ; mais de grandes différences existent entre les femelles ; certaines entrent périodiquement en chaleur pendant 2 à 3 jours, d'autres acceptent le mâle pendant des durées beaucoup plus longues pouvant excéder 20 jours (Büttner et Wienert, 1935 ; Moret, non publié).

Les observations faites par l'un de nous suggèrent que de nombreux facteurs externes peuvent intervenir dans le déclenchement de l'œstrus ; c'est pourquoi nous avons entrepris une étude du rôle d'un changement brutal de l'environnement sur l'apparition de l'œstrus chez la lapine nullipare.

\section{Matériel et méthode.}

a) Animaux. - L'expérience a porté sur 104 lapines nullipares de souches néozélandaise ou californienne, âgées de 4 mois et demi à 5 mois au moment de l'expérience. Les lapines étaient élevées en cage individuelle sur litière de paille en clapiers sifués à l'extérieur dans le groupe 1, tandis que dans le groupe 2 elles étaient élevées 
en cage sur grillage, sous $12 \mathrm{~h}$ de lumière par $24 \mathrm{~h}$, dans un bâtiment maintenu à une température d'au moins $15^{\circ} \mathrm{C}$.

Dans le groupe 1, l'expérience a été réalisée en novembre, c'est-à-dire en photopériode naturelle courte :

- 28 femelles constituant le lot expérimental ont été placées dans des cages sur grillage dans un bâtiment d'élevage sous $16 \mathrm{~h}$ de lumière par $24 \mathrm{~h}$ subissant ainsi une triple modification d'environnement : cage, durée d'éclairement et température. Parmi ces femelles, 6 ont subi des manipulations quotidiennes consistant en des prises de sang journalières une semaine avant et une semaine après les débuts des tests; - 14 femelles constituant le lot témoin ont été laissées sur litière de paille, dans les mêmes cages à l'extérieur ; 7 de ces femelles ont été manipulées comme décrit précédemment.

Dans le groupe 2 :

- 41 femelles constituant le lot expérimental ont été changées de bâtiment d'élevage et mises sous $16 \mathrm{~h}$ de lumière par $24 \mathrm{~h}$. Elles ont donc subi un double changement : cage et durée d'éclairement; 17 de ces lapines ont été manipulées comme dans le groupe 1 ;

- 22 femelles témoins ont été laissées dans leurs cages et n'ont donc subi aucun changement. Dix d'entre elles ont été manipulées comme dans le groupe 1.

b) Paramètres étudiés. - Le comportement d'œstrus a été déterminé en plaçant la femelle dans la cage d'un ou plusieurs mâles. Les mâles étaient logés dans les mêmes conditions que les lapines testées, à savoir sur litière quand les femelles étaient sur litière ef sur grillage quand elles étaient sur grillage et sous les mêmes durées d'éclairement.

Les présentations au mâle ont commencé 24 h après le changement d'environnement et ont été répétées 4 jours de suite jusqu'au jour de l'acceptation.

Les comparaisons entre les lots témoins et expérimentaux ont été faites à l'aide du test de $X^{2}$.

\section{Résultats.}

Dès le premier jour de présentation au mâle, une différence apparaît entre les lots. Les taux d'acceptation sont plus élevés dans les lots expérimentaux que dans les lots témoins. Un changement brutal d'environnement provoque donc une apparition rapide de l'œstrus chez la lapine nullipare. La différence est plus nette dans le groupe 2 $(P<0,01)$ que dans le groupe 1 (fig. 1 et tabl. 1).

Le nombre des femelles en œstrus est plus élevé parmi les femelles ayant subi des manipulations quotidiennes. La différence est significative si l'on considère l'ensemble des femelles : 50 p. 100 des femelles manipulées acceptent le mâle dès le premier jour ; 13 p. 100 seulement pour les femelles non manipulées $(P<0,01)$ (tabl. 1$)$.

Le nombre de femelles en œstrus augmente entre le 1 er et le $4 \mathrm{e}$ jour de présentation de 27 à 72 p. $100(P<0,01)$. Cette augmentation est particulièrement nette chez les femelles manipulées ou non du groupe $2(P<0,01)$. Au contraire, chez les femelles 
témoins du groupe 2, les taux d'acceptation n'augmentent pas significativement (fig. 1).
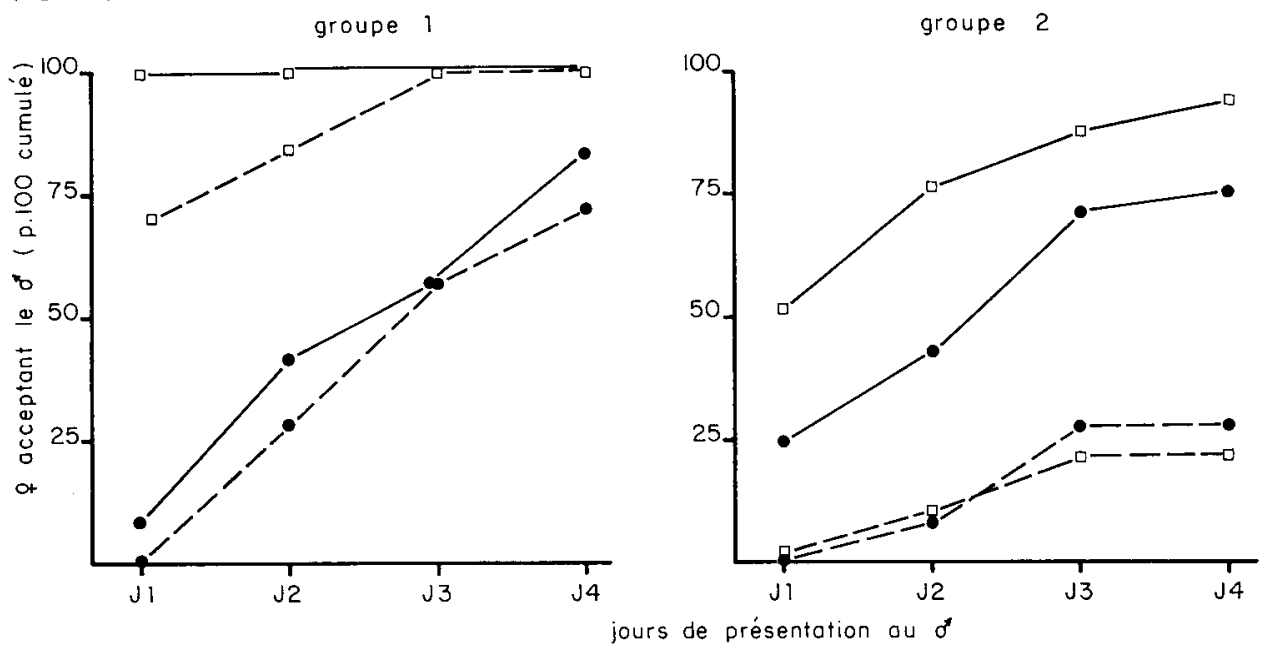

FIG. 1. - Apparition de l'œstrus chez les femelles des lots expérimentaux soumises à un changement d'environnement et chez les femelles témoins.

\begin{tabular}{|c|c|}
\hline - - femelles non manipulées & iental \\
\hline$\square \longrightarrow$ feme & ienral \\
\hline $\begin{array}{l}-\ldots \text { femelles non manipulées } \\
\square-\ldots \text { femelles manipulées }\end{array}$ & lot \\
\hline
\end{tabular}

\section{TABLEAU 1}

Taux d'acceptation du mâle

\begin{tabular}{|c|c|c|c|c|c|}
\hline & & \multirow{2}{*}{ Lot } & \multirow{2}{*}{$\begin{array}{l}\text { Nombre de } \\
\text { femelles }\end{array}$} & \multicolumn{2}{|c|}{$\begin{array}{c}\text { Femelles ayant accepté } \\
\text { l'accouplement }\end{array}$} \\
\hline & & & & $\begin{array}{l}\text { le } 1 \text { er jour } \\
(p .100)\end{array}$ & $\begin{array}{c}\text { En } 4 \text { jours } \\
(p .100)\end{array}$ \\
\hline Groupe $1 \ldots$ & $\begin{array}{l}\text { Témoin } \\
\text { Expérimental }\end{array}$ & $\begin{array}{l}\text { : manipulé } \\
\text { : non manipulé } \\
\text { : manipulé } \\
\text { : non manipulé }\end{array}$ & $\begin{array}{r}7 \\
7 \\
6 \\
22\end{array}$ & $\begin{array}{r}71 \\
0 \\
100 \\
9\end{array}$ & $\begin{array}{r}100 \\
71 \\
100 \\
82\end{array}$ \\
\hline Groupe $2 \ldots$ & $\begin{array}{l}\text { Témoin } \\
\text { Expérimental }\end{array}$ & $\begin{array}{l}\text { : manipulé } \\
\text { : non manipulé } \\
\text { : manipulé } \\
\text { : non manipulé }\end{array}$ & $\begin{array}{l}10 \\
11 \\
17 \\
24\end{array}$ & $\begin{array}{r}0 \\
0 \\
54 \\
25\end{array}$ & $\begin{array}{l}20 \\
27 \\
94 \\
75\end{array}$ \\
\hline
\end{tabular}

\section{Discussion.}

Un brusque changement d'environnement facilite donc l'apparition de l'œestrus chez la Lapine nullipare. Ce phénomène a déjà décrit chez d'autres espèces, la Truie notamment (du Mesnil du Buisson et Signoret, 1962). Ce travail présente donc un 
modèle expérimental facile pour obtenir chez des Lapines nullipares de 4 à 5 mois des femelles en cstrus.

La notion d'environnement est complexe ; quelques paramètres seulement peuvent être analysés à partir de ce travail.

La brusque modification de l'environnement peut agir simplement comme un stress en provoquant une décharge hormonale dans le sang périphérique. Le rôle positif des manipulations quotidiennes sur l'apparition de l'œstrus confirme cette hypothèse.

La modification de la photopériode intervient peut-être non en tant que stress mais en tant que durée d'éclairement. L'augmentation de la durée d'éclairement subie par les lapines, passage d'une photopériode claire quotidienne de novembre pour le groupe 1 , de $12 \mathrm{~h}$ pour le groupe 2 à une photopériode claire de $16 \mathrm{~h}$ par $24 \mathrm{~h}$, doit avoir un rôle favorable sur l'activité ovarienne puisque les femelles en chaleur sont plus nombreuses au printemps et en été qu'en automne (Bradbury, 1944). Chez l'Oiseau, une augmentation brutale de la photopériode claire quotidienne provoque une augmentation de la LH plasmatique dans les $24 \mathrm{~h}$ qui suivent (Follet et al., 1975).

La stimulation provoquée par des présentations successives au mâle permet de penser que les stimuli provenant du mâle facilitent l'apparition de l'œstrus. Ces stimuli sont vraisemblablement d'ordre olfactif ; en effet, chez les femelles du groupe 1 élevées sur litière et présentées à des mâles vivants aussi sur des litières de paille, on observe un pourcentage de femelles en cestrus très important dès le 1 er jour et une augmentation de ce pourcentage très rapide au cours des 4 jours. Les litières de paille doivent retenir beaucoup plus les odeurs d'urine que les cages en grillage ef le travail de Franck (1966) a montré l'importance des stimuli olfactifs sur la maturation sexuelle de la Lapine.

Reçu en octobre 1977.

Accepté en jonvier 1978.

\section{Références}

BRADBURY J. T., 1944. Seasonal incidence of anoestrus in the postpartum rabbit. Anat. Rec., 88, 424426.

BUTTNER W., WIENERT K., 1935. Dauerbrunst und Follikelpersistenz-Experimentelle Untersuchungen an Kaninchen. Arch. Gynak., 159, 64-83.

FOLLET B. K., FARNER D. S., MATTOCKS P. W., 1975. Luteinizing hormone in the plasma of white crowned sparrow during artificial photostimulation. Gen. comp. Endocrinol., 26, 126-134.

FRANCK H., 1966. Ablation des bulbes olfactifs chez la lapine impubère. Répercussions sur le tractus génital et le comportement sexuel. C. R. Soc. Biol., 160, 389-390.

HAMMOND J. R., MARSHALL F. H. A., 1925. Reproduction in the rabbit. Oliver and Boyd, Edinburgh, $210 p$.

HEATH E., 1972. Sexual and related territorial behavior in the laboratory rabbit (Oryctolagus cuniculus). Lab. Anim. Sci., 22, 684-691.

HILL M., WHITE W., 1933. The growth and regression of follicles in the cestrous rabbit. J. Physiol., 80, 174-178.

MESNIL du BUISSON F. du, SIGNORET J. P., 1962. Influence de facteurs externes sur le déclenchement de la puberté chez la truie. Ann. Zootech., 11, 53-59.

MYERS K., POOLE W. E., 1962. Estrus cycles in the rabbit, Oryctolagus cuniculus. Nature, 195, 358-359.

SMELSER G. R., WALTON A., WHETHAM E. D., 1934. The effect of light on ovarian activity in the rabbit. J. exp. Biol., 11, 352-363. 